\title{
Category vs. item learning: Implications for categorization models
}

\author{
STEPHEN K. REED \\ Case Western Reserve University, Cleveland, Ohio 44106
}

\begin{abstract}
A paradigm that required that subjects learn two responses to each of 10 schematic faces was used to study the relative rate of discrimination and generalization learning. One response uniquely identified each face, whereas the second response classified each face as a member of one of two categories. Rapid category learning and slow item learning suggested that category responses were learned on the basis of abstracted information, but item responses depended on the more difficult task of discriminating among patterns. The results are related to categorization models and to task variables that should influence the relative rates of discrimination and generalization learning.
\end{abstract}

There have been two general approaches to the study of pattern recognition and perceptual learning. One approach has viewed pattern recognition as a problem of discrimination learning (Gibson \& Gibson, 1955), whereas the other approach has viewed pattern recognition as a problem in learning to make appropriate categorizations (Bruner, 1957). Gibson and Gibson argued that perceptual learning occurs through the progressive elaboration of features or dimensions of variation. Perceptual learning involves responding to variables of physical stimulation not previously responded to, so that perception becomes richer in differential responses, not in images. In contrast, Bruner argued that perception is the end product of a categorization process, that whatever is perceived achieves its meaning from the class of percepts with which it is grouped. Bruner believed that the rules for making perceptual classifications are basically the same as the rules for making more conceptual inferences. The rules should specify (1) the critical properties of the stimuli, (2) the manner in which the properties are combined, (3) the weights assigned various properties, and (4) the acceptance limits for each category.

The two theories have different implications for how people view the world. According to a discrimination theory, many objects look the same initially, and the observer has to learn to perceive how they differ. According to a categorization theory, objects look different from other objects, and the observer has to make an appropriate generalization. Theories, of course, are usually tested in the laboratory, and the reason these two theories have seldom met "head on" in the laboratory is that each theory has given rise to a different kind of experiment. ${ }^{1}$

I am grateful to Jeff Johnsen and Mark Westerhaus for their assistance on this project. Requests for reprints should be sent to Stephen K. Reed, Department of Psychology, Case Western Reserve University, Cleveland, Ohio 44106.
The experiment performed by Gibson and Gibson (1955) is typical of the kind of paradigm used by investigators of discrimination learning (see E. Gibson, 1969, for other examples). The stimuli were nonsense forms that differed from a standard form along three dimensions of variation: number of coils, horizontal stretching, and right-left reversal. Subjects viewed the standard form for $5 \mathrm{sec}$ and were told that some patterns in a pack of cards exactly matched the standard. They then looked at the cards, one at a time, and selected those patterns that they thought were identical to the standard. Since patterns were compared to a single exemplar, categorization was not necessary.

Experiments on categorization learning have usually been conducted by investigators interested in the abstraction of prototypes. The experiment by Posner and Keele (1968) is a typical example. Categories were formed by distorting dot patterns that formed a triangle, the letters $M$ and $F$, and a random dot pattern. Subjects learned a list of 12 patterns that included 3 from each category. Since patterns from the same category had the same response, both generalization and discrimination are involved: generalization among patterns belonging to the same category and discrimination between patterns belonging to different categories. In a pattern classification task, subjects classified 24 new patterns into one of the four categories they had learned during the initial learning task. Classification of new patterns presumably requires some rule of induction, as proposed by Bruner (1957).

The following research is based on a paradigm that attempts to study the simultaneous occurrence of discrimination and generalization leaming. The task required that subjects learn two responses to each of the 10 schematic faces shown in Figure 1. One response is a category response that identifies each face as belonging to either Category A (upper five faces) or to Category B (lower five faces). The other response is a number (from 1 to 10 ) that identifies each individual 


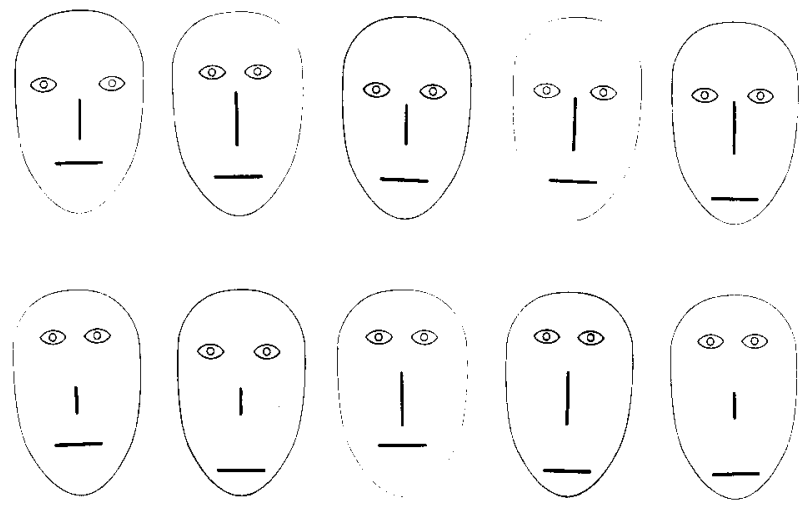

Figure 1. Two categories of schematic faces. Category A faces are in the upper row and Category B faces are in the lower row. The faces are identified in Tables 1 and 2 by the numbers 1-10. starting with the left face in the upper row and ending with the right face in the bottom row.

face. The subject therefore has to learn to discriminate all 10 faces, in addition to learning which 5 faces belong to a common category.

One reason for using this paradigm is that it seems plausible that perceptual learning outside the laboratory involves a continual interplay between discrimination and generalization learning. Discrimination leaming is required to initially establish different categories, but once established, many subsequent patterns can be recognized by an act of induction. The degree of perceptual similarity among objects is a prime determinant of the extent to which discrimination or generalization is involved. A child's two pet calves may look so much alike that considerable discrimination learning is necessary to tell them apart, but two dogs may look so different that an act of categorization is necessary to establish them as members of the same category. The demands of the task are also prime determinants of the extent to which discrimination or generalization is necessary. When reading someone's handwriting, it is usually only necessary to classify letters by identifying the appropriate letter category (is that an a or an o?). However, handwriting experts must also be able to discriminate among patterns within a category (identifying different styles of the letter a) in order to determine whether two messages were written by the same person.

\section{CATEGORIZATION MODELS}

A more specific reason for conducting this research is that it should tell us something about the inference rules used by people to classify patterns. In particular, different categorization rules differ in the extent to which they require discrimination among the patterns within a category. For example, Reed (1972) compared four different models to determine which model would be most successful in predicting how people would categorize patterns. Each task consisted of two cate- gories containing five schematic faces. Figure 1 shows the two categories used in one of the tasks (Reed, 1972, Problem 2A).

According to the "average distance" model, subjects should choose the category containing patterns that are the least average distance from the test pattern. This strategy requires that subjects compare the distance of the test pattern to each of the five patterns in the two categories and compute the average distance of the test pattern from each category. Another model that requires comparing the distance of the test pattern from each category pattern is the "nearest neighbor" (closest match) rule. According to this strategy, the subject should choose the category containing the pattern that is the least distant from the test pattern. Since the average distance model and nearest neighbor model require discrimination among the members within a category, these models can be rejected in those cases in which subjects are unable to make within-categories discriminations. Learning the correct category response much more quickly than learning the correct item response would suggest that category responses are learned on the basis of some abstraction (features or prototypes), rather than through discrimination leaming of the individual exemplars.

The alternative is that subjects do not learn to classify patterns by learning the individual exemplars, but instead learn to classify patterns on the basis of some abstraction. One example is the "prototype" model, which assumes that subjects create a pattern to represent the central tendency of each category. The advantage of a prototype model is that it makes about the same kind of decisions as the average distance model, but does not require memory for the individual exemplars. ${ }^{2}$ All that is required is memory for the category prototype and the number of patterns previously classified into that category. A change in the prototype can be expressed by Equation 1, in which $P$ represents the feature values of the prototype, $X$ represents the feature values of a new category pattern, and $N$ represents the total number of patterns in the category, including $X$ :

$$
\text { New Prototype }=\mathrm{P}+1 / \mathrm{N} \cdot(\mathrm{X}-\mathrm{P}) \text {. }
$$

Equation 1 states that, when a new category pattern is perceived, the prototype moves toward the new category pattern, but how far it moves is inversely proportional to the number of patterns in the category. When the prototype is based on many previously seen patterns, it is influenced very little by a new category pattern.

The prototype model predicts that the ease of learning a pattern should be a function of how far it is from the two category prototypes. Ease of learning should be a function of the pattern's distance from the incorrect prototype minus its distance from the correct prototype. A special case of this model, the weightedfeatures prototype model, differentially weights the 
features of a pattem when computing distances, emphasizing those features that best discriminate between the two categories. The weights are calculated by using a class-separating transformation proposed by Sebestyen (1962). The linear transformation maximizes between-categories distances while holding constant the sum of between- and within-categories distances. For the categories shown in Figure 1, the weights are .46 for foreh'ad, .24 for eye separation, .24 for nose length, and .06 for mouth height.

Another model that does not require discrimination learning within a category utilizes feature fiequencies. The "frequency" model proposes that subjects classify test patterns by determining the relative frequency with which the features of the test pattern appear in the two categories. If there are an equal number of patterns in the two categories, the decision rule is to choose the category that results in the greatest number of feature matches. It is not necessary to learn the individual pattems in a category because only the distribution of features is used in the decision. A person using this strategy for the categories shown in Figure 1 would have to know the number of times a short, medium, and long nose appeared in each category, plus the distribution for each of the other three features.

A number of investigators (Hayes-Roth \& HayesRoth, 1977; Neumann, 1974; Reitman \& Bower, 1973) have proposed models based on frequency distributions. In addition to using frequencies of the individual features, these investigators have proposed using frequencies of combinations of features. For example, the "n-gram" model considered by Reitman and Bower calculates the number of matches for single features, all possible pairs of features, all possible triples of features, and so on. Predictions of both the simple frequency model, based on individual features, and the n-gram model, based on all combinations of features, are derived by calculating the number of feature matches with patterns in the correct category, as opposed to patterns in the incorrect category. The models predict that the ease of learning a pattern should be related to the number of correct category feature matches minus the number of incorrect category feature matches. The frequency model uses only individual features in calculating matches, whereas the n-gram model includes all possible combinations of pairs and triples of features, in addition to the individual features.

As mentioned previously, the learning paradigm requires that subjects learn two responses for each of the 10 faces. One approach is to randomly assign numbers to letters and have subjects give both responses on each trial, such as "A3" or "B7." The problem with this approach is that subjects probably learn the letter-number pairings before leaming the faceletter or face-number pairings, making it impossible to study generalization and discrimination learning independently. In order to prevent association between numbers and letters, subjects could alternate between letter and number responses, giving letter responses on odd-numbered trials and number responses on even-numbered trials. This procedure was used in Experiments 3 and 4 . In another version of the paradigm, used in Experiment 2, subjects gave both a letter and a number response on each trial, but were informed that faces in Category A were numbered from 1 to 5 and faces in Category B were numbered from 6 to 10 . There is a correlation between responses in this case (e.g., knowing the correct number indicates the correct letter), but the letter-number associations are given at the outset, making it easier to correct for guessing. The advantage of requiring both responses on each trial is that twice as much information can be collected over the same number of trials. The standard procedure, used in Experiment 1, required that subjects only learn category responses.

\section{EXPERIMENT 1}

The first experiment required only category learning and involves an analysis of previously collected data (Reed, 1972, Problem 3). Subjects were asked to learn the correct category responses for each of the 10 faces shown in Figure 1. They were not required to learn any item responses, but were asked to classify 24 test patterns after the learning experiment. Like most other research on categorization, interest initially focused on a subject's classifications and the leaming data were ignored. The learning data are analyzed here in order to determine the difficulty of learning a category response to each face when an item response is not required. Although it is not possible to compare the rate of learning with the other three experiments because of differences in subjects and procedure, the relative difficulty of learning the correct category response across faces should be informative.

\section{Method}

Subjects were 50 undergraduates at the University of California, Los Angeles, who were tested in small groups. They were enrolled in an introductory psychology course and received course credit for participating. Each face was projected at the front of a classroom for 6 sec. Subjects responded by circling one of two numbers, depending on which category they thought was correct. Two lights at the front of the room provided feedback regarding the correct response. Feedback was given during the last 2 sec of the 6-sec interval, and subjects were instructed to respond during the first $4 \mathrm{sec}$, guessing when uncertain. There was a 6 -sec intertrial interval (ITI). Subjects were told to look for similarities among faces belonging to the same category and were informed that the faces would differ along four dimensions: the height of the eyes, the distance between the eyes, the length of the nose, and the height of the mouth. The learning experiment consisted of 13 trials for each face. The order of presentation was organized into 13 successive blocks, with each block consisting of the 10 faces presented in a random order.

\section{Results}

Table 1 shows the average error probability for each of the 10 faces. The faces are identified by the numbers $1-10$, starting with the top row in Figure 1 and proceeding from left to right. Error probabilities were 
Table 1

Category Error Probabilities and Model Correlations

\begin{tabular}{|c|c|c|c|c|c|c|c|}
\hline & \multicolumn{6}{|c|}{ Experiment } & \multirow{4}{*}{$\begin{array}{c}\text { Aver- } \\
\text { age }\end{array}$} \\
\hline & \multicolumn{2}{|c|}{1} & \multicolumn{2}{|c|}{2} & \multicolumn{2}{|c|}{3} & \\
\hline & \multicolumn{2}{|c|}{ Trials } & \multicolumn{2}{|c|}{ Trials } & \multicolumn{2}{|c|}{ Trials } & \\
\hline & $1-7$ & $8-13$ & $1-8$ & $9-15$ & $1-5$ & $6-10$ & \\
\hline Face 1 & .11 & .07 & .14 & .05 & .20 & .17 & .12 \\
\hline Face 2 & .36 & .28 & .55 & .41 & .52 & .54 & .44 \\
\hline Face 3 & .16 & .08 & .17 & .08 & .23 & .14 & .14 \\
\hline Face 4 & .10 & .04 & .22 & .07 & .27 & .13 & .14 \\
\hline Face 5 & .17 & .13 & .26 & .16 & .34 & .21 & .21 \\
\hline Face 6 & .10 & .10 & .22 & .14 & .20 & .10 & .14 \\
\hline Face 7 & .30 & .21 & .30 & .20 & .29 & .25 & .26 \\
\hline Face 8 & .35 & .23 & .33 & .13 & .35 & .15 & .26 \\
\hline Face 9 & .12 & .01 & .17 & .08 & .24 & .09 & .14 \\
\hline Face 10 & .06 & .01 & .16 & .09 & .23 & .07 & .10 \\
\hline Model 1 & .73 & .70 & .74 & .65 & .75 & .83 & .73 \\
\hline Model 2 & .67 & .70 & .65 & .68 & .65 & .80 & .69 \\
\hline Model 3 & .82 & .89 & .73 & .74 & .66 & .62 & .74 \\
\hline Model 4 & .80 & .84 & .83 & .85 & .71 & .78 & .80 \\
\hline
\end{tabular}

Note-Model numbers refer to the (1) frequency, (2) n-gram, (3) prototype, and (4) weighted-features prototype models.

calculated for the first seven and last six learning trials. The data indicate that there were substantial differences in the ease of learning the correct category response across the 10 faces. The average error probability ranged from .06 to .36 over the first seven learning trials and from .01 to .28 over the last six learning trials.

The relative difficulty of learning the correct category response was correlated with the predicted difficulty as determined by the feature frequency, n-gram, prototype, and weighted-features prototype models. The prototype model made the most accurate predictions, producing a correlation of .89 over the last six trials (see Table 1). All correlations were significant at least at the $p=.05$ level, and none of the models predicted significantly better than the others. The next experiment required both category learning and item learning.

\section{EXPERIMENT 2}

\footnotetext{
Method

The 62 subjects in Experiment 2 were undergraduates at Case Western Reserve University. They were enrolled in psychology courses and received course credit for their participation. The procedure was similar to that used in Experiment 1. Faces were shown for $7 \mathrm{sec}$, with oral feedback given at the end of $4 \mathrm{sec}$. The ITI was $6 \mathrm{sec}$. The 10 faces were presented in a random order within each block of 10 trials. The response sheets were not numbered, in order to prevent subjects from eliminating responses that occurred earlier in a block. The division into blocks was therefore not evident, and the experiment appeared continuous to the subject. The instructions indicated that the subjects would have to learn two responses to each face: a number response (from 1 to 10 ) identifying each face and a category response indicating which five faces belonged to Category $A$ and which five faces belonged to Category B. As in the previous experiment, subjects were told which four features would vary and were asked to look for similarities among faces belonging to the same category.
}

Subjects in Experiment 2 gave both responses on each trial. They were told that faces in Category A were numbered from 1 to 5 and faces in Category $B$ were numbered from 6 to 10. The responses were therefore $\mathrm{A} 1, \mathrm{~A} 2, \mathrm{~A} 3, \mathrm{~A} 4, \mathrm{~A} 5, \mathrm{~B} 6, \mathrm{~B} 7, \mathrm{~B} 8, \mathrm{~B} 9$, and $B 10$. In order to limit the duration of the experiment to $50 \mathrm{~min}$, there was a total of 150 presentations $(15$ trials $\times 10$ faces). The data from six subjects were not included in the analysis because they failed to give a response on 20 or more presentations. For the remaining 56 subjects, the average number of missed responses was $2.1 \%$. A random-number generator was used to assign a response for these cases.

\section{Results}

Figure 2 shows the probability of making an item error and a category error over the 15 learning trials. Table 2 shows the probability of giving the wrong category response for each of the 10 faces. The average probability of an error is shown separately for the first eight trials and the last seven trials. The weightedfeatures prototype model made the most accurate predictions regarding the relative difficulty of the 10 faces. The correlation between error probability and predicted difficulty was .83 for the first eight trials and .85 for the last seven trials. However, the correlations of all models were significant at least at the $p=.05$ level, and no one model predicted significantly better than the others.

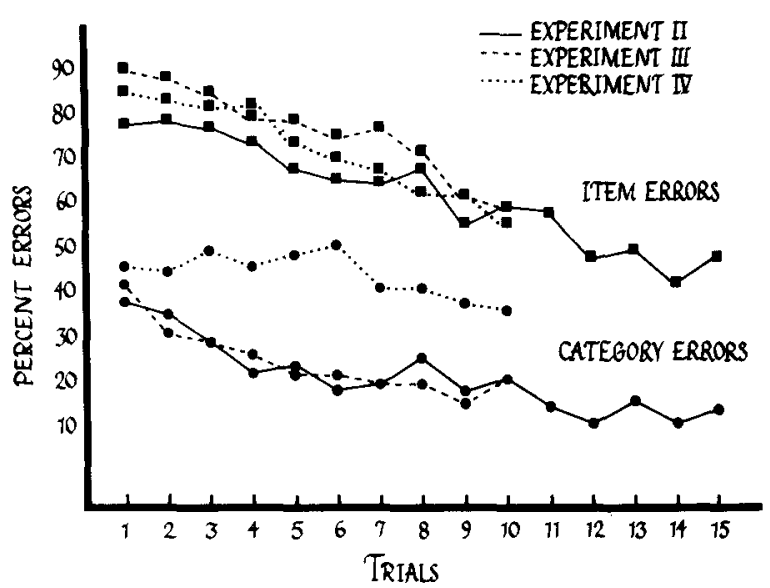

Figure 2. Learning curves for category and item responses.

Table 2

Average Probability of an Item Error for Each Face

\begin{tabular}{|c|c|c|c|c|c|c|}
\hline \multirow[b]{2}{*}{ Face } & \multicolumn{2}{|c|}{ Experiment 2} & \multicolumn{2}{|c|}{ Experiment 3} & \multicolumn{2}{|c|}{ Experiment 4} \\
\hline & Response & Errors & Response & Errors & Response & Errors \\
\hline 1 & $5 \mathrm{~A}$ & .47 & $10 \mathrm{~A}$ & .65 & $10 \mathrm{~A}$ & .64 \\
\hline 2 & $4 \mathrm{~A}$ & .74 & $8 \mathrm{~A}$ & .80 & $8 \mathrm{~A}$ & .76 \\
\hline 3 & $3 \mathrm{~A}$ & .58 & $7 \mathrm{~A}$ & .79 & $7 \mathrm{~A}$ & .78 \\
\hline 4 & $2 \mathrm{~A}$ & .65 & $3 \mathrm{~A}$ & .78 & $3 \mathrm{~B}$ & .74 \\
\hline 5 & $1 \mathrm{~A}$ & .63 & $1 \mathrm{~A}$ & .78 & $1 \mathrm{~B}$ & .60 \\
\hline 6 & $7 \mathrm{~B}$ & .61 & 4B & .72 & $4 B$ & .72 \\
\hline 7 & $6 \mathrm{~B}$ & .68 & $2 \mathrm{~B}$ & .77 & $2 B$ & .70 \\
\hline 8 & $10 \mathrm{~B}$ & .65 & $9 \mathrm{~B}$ & .78 & $9 \mathrm{~B}$ & .73 \\
\hline 9 & $9 \mathrm{~B}$ & .50 & $6 \mathrm{~B}$ & .78 & $6 \mathrm{~A}$ & .77 \\
\hline 10 & $8 \mathrm{~B}$ & .62 & $5 \mathrm{~B}$ & .77 & $5 \mathrm{~A}$ & .72 \\
\hline
\end{tabular}


Table 2 shows the average probability of an item error for each of the 10 faces. The results support the expected correlation between item learning and category learning when subjects gave both responses on each trial. Since the numbers $1-5$ are associated with Category $A$ and 6-10 are associated with Category $B$, learning the correct category should increase the probability of guessing the correct number. Faces 1 and 9, which are relatively easy; to categorize (see Table 1 ), have the lowest error rate. Face 2, which is difficult to categorize, has the highest error rate because subjects often guessed a Category B number. A significant correlation $[r(8)=.78, p<.01]$ was obtained between the probability of a category error and the probability of an item error for each of the 10 faces. Experiment 3 attempted to separate category and item responses by randomly assigning numbers to letters and requiring only a single response (category or item) on each trial.

\section{EXPERIMENT 3}

\section{Method}

The procedure used for Experiment 3 was identical to the procedure used for Experiment 2 except that only a single response was required on each trial. Subjects gave category responses on odd-numbered trials and item responses on evennumbered trials. The numbers $1,3,7,8$, and 10 were randomly assigned to Category $A$. Table 2 shows the letter and number assigned to each face. There were a total of 200 presentations (10 trials $\times 2$ responses $\times 10$ faces), and the experiment required about $50 \mathrm{~min}$ to complete.

The data from 4 of the 50 subjects were not used because these subjects gave no response on 20 or more presentations. The average number of missed responses was $1.8 \%$ for the remaining subjects. A randomly chosen response was assigned for these cases. Subjects were undergraduates at Case Western Reserve who received course credit for participating.

\section{Results}

Table 1 shows the category error probabilities for each of the 10 faces. The feature frequency model best predicted the relative difficulty of learning the correct category responses. The correlation was .83 over the last five learning trials. However, the predictions of this model were not significantly better than the predictions of the other three models.

Figure 2 shows the probability of making an item error and a category error over trials. The learning curves for the category responses were virtually identical in Experiments 2 and 3 , suggesting that the rate of category learning was unaffected by the item learning occurring on every other trial. For each category trial, subjects in Experiment 3 had seen a face twice as often as subjects in Experiment 2, but this additional opportunity to discriminate among the faces on item trials did not improve category leaming. The possible implication is that category learning and item learning occur independently, with category learning based on some abstraction that does not depend on discrimination among the exemplars. In order to investigate this hypothesis, it is necessary to determine when item learning and category learning occur.

The finite-integer model proposed by Theios (1968) was used to determine whether a sequence of responses was more likely to come from an unleamed or a leamed state. Let us assume that the probability of a correct response after category or item learning is .95. This requires a high performance level but allows for an occasional error. Let us further assume that the probability of a correct response prior to learning is the initial probability of guessing correctly, .5 in the case of a category response and .1 in the case of an item response. Consider now a sequence of independent responses containing $\mathrm{C}$ correct responses and $\mathrm{W}$ wrong responses. For category and item learning, the probability of a particular sequence, given that learning has occurred, is $(.95) \mathrm{C}(.05)^{\mathrm{W}}$. The probability of that sequence, given that learning has not occurred, is $(.1) \mathrm{C}(.9)^{\mathrm{W}}$ for item responses and $(.5)^{\mathrm{C}}(.5)^{\mathrm{W}}$ for category responses.

Theios's (1968) model involves finding a trial number, $\dot{i}$, that maximizes the likelihood that responses prior to $i$ come from an unlearned state and responses following $\mathrm{i}$ come from a learned state. For category learning, the likelihood is given by the product of $(.5)^{\mathrm{c}}(.5)^{\mathrm{w}}(.95)^{\mathrm{C}}(.05)^{\mathrm{W}}$, where $\mathrm{c}$ is the number of correct responses prior to $\mathrm{i}, \mathrm{w}$ is the number of wrong responses prior to $\mathrm{i}, \mathrm{C}$ is the number of correct responses following (and including) $\mathrm{i}$, and $\mathrm{W}$ is the number of wrong responses following $i$. For item learning, the likelihood is given by the product of $(.1)^{\mathrm{c}}(.9)^{\mathrm{w}}(.95)^{\mathrm{C}}(.05)^{\mathrm{W}}$. If we now consider a sequence of responses containing one error, a minimum of four correct category responses is necessary to make it more likely that the sequence came from a learned state, since $(.95)^{4}(.05)^{1}>(.5)^{4}(.5)^{1}$, but $(.95)^{3}(.05)^{1}<(.5)^{3}(.5)^{1}$. But only two correct item responses are necessary, since $(.05)^{2}(.05)^{1}>(.1)^{2}(.9)^{1}$. I therefore used the criteria that category leaming could not occur later than Trial 7 (which resulted when the last four responses were correct and previous responses were from the unlearned state), and item learning could not occur later than Trial 9 (which resulted when the last two responses were correct and previous responses were from the unleraned state). Category or item learning was determined to have occurred on an earlier trial if it maximized the appropriate likelihood expression.

Table 3 shows for each of the 550 faces in Experiment 3 (55 subjects $X 10$ faces) the trial on which learning occurred, using the above criteria. Both responses were leamed for 148 faces, only a category response was learned for 271 faces, only an item response was leamed for 27 faces, and neither response was leamed for 104 faces. The superiority of category learning is quite evident from these results. At the end of the 10 trials, correct category responses had been learned for $76 \%$ of the faces, but correct item responses had been learned for only $32 \%$ of the faces $(z=14.14$, $\mathrm{p}<.001$ ). Of the 148 cases in which both responses 
Table 3

Number of Category Responses and Item Responses Learned on Each Trial in Experiment 3

\begin{tabular}{|c|c|c|c|c|c|c|c|c|}
\hline \multirow{2}{*}{$\begin{array}{l}\text { Item } \\
\text { Learn- } \\
\text { ing } \\
\text { Trials }\end{array}$} & \multicolumn{8}{|c|}{ Category Learning Trials } \\
\hline & 1 & 2 & 3 & 4 & 5 & 6 & 7 & $\mathbf{N}$ \\
\hline 1 & 1 & & & & & & & \\
\hline 2 & 3 & & & & & 1 & & \\
\hline 3 & 4 & 1 & 2 & 1 & 1 & 1 & & 1 \\
\hline 4 & 5 & 4 & 1 & 1 & 1 & & & \\
\hline 5 & 7 & 3 & 4 & & 1 & & & 3 \\
\hline 6 & 7 & 2 & 4 & 2 & 1 & & & 1 \\
\hline 7 & 6 & 2 & 5 & 2 & 3 & & & 6 \\
\hline 8 & 14 & 14 & 5 & 4 & 2 & 4 & 1 & 12 \\
\hline 9 & 12 & 3 & 1 & 5 & 5 & 1 & 1 & 4 \\
\hline $\mathbf{N}$ & 112 & 44 & 26 & 30 & 28 & 23 & 8 & 104 \\
\hline
\end{tabular}

Note $-N$ indicates the number of unlearned responses. The sum of each column is the total number of category responses learned on that trial; the sum of each row is the total number of item responses learned on that trial. Each entry is the number of cases in which a category response and an item response were learned on that particular pair of trials. For example, there were 112 cases in which a category response was learned on the first trial and an item response was never learned.

were learned, the item response was learned first in only five cases. Figure $3 a$ shows the percent of faces for which a category response or an item response was learned on each trial. In contrast to the item responses, many of the category responses were learned very quickly, including the correct response to $31 \%$ of the faces on the first trial.

In addition to the fact that category learning occurs much more rapidly than item learning, the results indicate that item learning and category learning are relatively independent of each other. It should first be noted that, with the exception of Face 1, all faces in Experiment 2 were about equally difficult to associate with the correct item response (Table 2). In contrast, there was a wide range among faces in difficulty of learning the appropriate category response (Table 1 ). A nonsignificant correlation $[\mathrm{r}(8)=.39]$ was obtained between the average probability of a category error and the average probability of an item error for the 10 faces. Further evidence for the weak relation between category and item learning is the low correlation between the trials on which item learning and category learning occurred. This correlation was $.13(\mathrm{p}>.05)$ for the 148 faces for which both responses were learned. My interpretation of these results is that category learning occurs much more rapidly than item learning because subjects learn some abstraction (such as a category prototype or critical features) that enables them to classify patterns before they can discriminate the exemplars from each other. Since discrimination learning requires different and more detailed information than category learning, it occurs more slowly than category learning.

\section{Discussion}

These results have several implications. First, they demonstrate that, for the categories used here, some form of abstraction allows for a fairly rapid learning of category responses that is independent of the slower discrimination learning required to identify individual exemplars. Second, there were still many unlearned item responses at the end of the experiment, suggesting that discrimination learning was far from complete. Since the number of learning trials (13) in my previous work on pattern classification (Reed, 1972) was within the range of the number of learning trials used here (10-15), it is doubtful whether there is enough within-categories discrimination of patterns to use a classification rule that requires comparing the distance of a test pattern to the individual exemplars. Furthermore, since category learning is much faster than item learning, and because item learning has not been required in classification experiments, there would be no incentive to attempt within-categories discrimination of patterns.

These results are therefore inconsistent with categorization models that postulate that novel patterns are compared to individual exemplars. Both the average distance and nearest neighbor models postulate such comparisons and would not seem very viable whenever discrimination learning within a category is as poor as that found in these experiments. The results are consistent with models that postulate abstraction of category information.

Four models of abstraction-a feature frequency model, an n-gram model, a prototype model, and a weighted-features prototype model-were compared to determine how successfully each could predict the relative difficulty of learning the correct category response to each of the 10 faces. The last column in Table 1 shows the average correlation coefficient for each model, computed by averaging the six values calculated for the first three experiments. The weightedfeatures prototype model made the most successful predictions across the three experiments, supporting

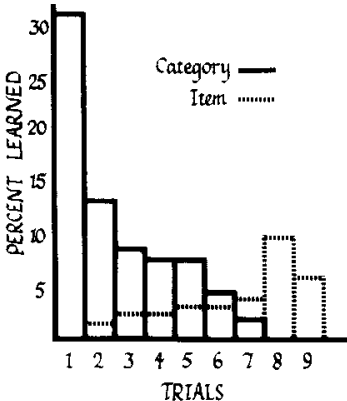

(a)

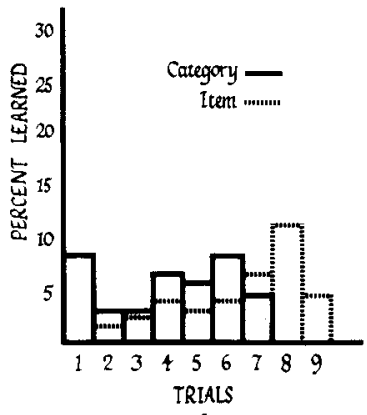

(6)
Figure 3. Percent of category and item responses learned on each trial for (a) Experiment 3, which used two categories shown in Figure 1, and (b) Experiment 4, which used two "random" categories. 
previous findings that indicated this model is also the most successful in predicting how people classify novel patterns (Reed, 1972).

Although the weighted-features prototype model is the best predicting model, all models are fairly capable of predicting learning difficulty. However, previous research indicated that the cue validity model did very poorly in predicting how people classify novel patterns (Reed, 1972; Reed \& Friedman, 1973). The selection of test patterns that were classified differently by the different models made it easier to discriminate among the models. It was anticipated that the frequency and $\mathrm{n}$-gram models would do poorly in predicting the classification of novel patterns because of their similarity to the cue validity model. This expectation was confirmed when these models were applied to the Reed (1972) and Reed and Friedman (1973) classification data. The predictions of the simple frequency and n-gram models were essentially the same as the predictions of the cue validity model.

At the end of Experiments 2 and 3, subjects were asked how they learned to distinguish between the two categories. Their answers were quite consistent with the concept of the weighted-features prototype model. There were 39 references to holistic or Gestalt characteristics, 28 to forehead height, 20 to eye separation, 20 to the distance between the eyes and nose, 10 to the nose, 7 to the distance between the nose and mouth, 5 to the distance between the eyes and mouth, and 5 to the mouth. ${ }^{3}$ References to Gestalt characteristics included such comments as "Category $\mathbf{A}$ faces are more oval, realistic, compact, and pleasant, whereas Category B faces are more harsh, extreme, and disjointed." These comments were quite frequent and seem to describe the typical pattern (prototype) representing each category. However, it is also clear that people emphasize some features more than others. According to the derived weights, the forehead or eye height should be the most important, eye width and nose length should be of intermediate and equal importance, and mouth height should be of least importance. The number of references to individual features was 28 to forehead, 20 to eyes, 10 to nose, and 5 to mouth. If feature combinations are included, there were 53 references to forehead, 37 to nose, 20 to eyes, and 17 to mouth. When feature combinations are included, eye separation is referred to less often than expected because it varies along the horizontal axis, whereas the other three features vary along the vertical axis. But the overall pattem of results supports the predictions: most references to the forehead, an intermediate number to the eyes and nose, and least references to the mouth.

\section{EXPERIMENT 4}

The interpretation of the results obtained in Experiment 3 was that item learning occurs more slowly than category learning because it requires different and more detailed stimulus information. One might argue, however, that item learning occurs more slowly, not because of the difficulty of discriminating among the patterns, but because of the difficulty of associating 1 of 10 responses to a pattern, rather than 1 of 2 responses. Experiment 4 was designed to test this interpretation by using the same paradigm as in Experiment 3 , with the modification that there was no relation among the five faces that shared a common response. The discrimination hypothesis would predict that the rate of learning the correct letter and number should now be approximately the same, since there is no basis for the abstraction of category information. The association hypothesis would predict that the learning results should be similar to Experiment 3, since the same associations are required.

\section{Method}

The procedure for Experiment 4 was identical to Experiment 3 , except that two faces from Category A were exchanged with two faces from Category B (the last two faces from each row in Figure 1) to create two categories that had nearly identical prototypes and feature distributions. It was expected that, when corrections for chance responding were made, the rate of category learning should be approximately the same as the rate of item learning because there was no basis for distinguishing between the two categories except by learning the individual exemplars. Subjects in Experiment 4 were told that both letters and numbers were randomly assigned to faces, and that there was no relation among faces associated with the same letter. Table 1 shows the letter and number assigned to each face.

There were 51 subjects who participated in the experiment, but the results from 7 subjects were not used because they gave no response on 20 or more presentations. The average number of missed responses was $2.5 \%$ for the remaining subjects. All subjects were undergraduates at Case Western Reserve who received course credit for participating.

\section{Results}

Figure 2 shows the results. Item learning proceeded at the same rate as in Experiment 3, but the rate of category learning was dramatically reduced when there was no basis for abstraction. In fact, there seemed to be very little category learning at all over the first six trials. These results occurred even though subjects were fully informed that letters and numbers were both randomly assigned to faces. When asked at the end of the experiment whether it was more difficult to learn letters or numbers, 25 subjects reported letters were more difficult, 16 subjects reported numbers were more difficult, and 3 subjects expressed no preference.

Theios's (1968) finite-integer model was again used to determine the trial on which item and category learning occurred. These results are shown in Table 4 . In contrast to Experiment 3, there was no significant difference between the percent of item and category responses learned: $42 \%$ for category responses and 38\% for item responses $(\mathrm{z}=1.23, \mathrm{p}>.05)$. However, category learning again occurred more quickly than item learning; of the 91 faces for which both responses were learned, the category response was learned first for 67 faces, the 
Table 4

Number of Category Responses and Item Responses Learned on Each Trial in Experiment 4

\begin{tabular}{|c|c|c|c|c|c|c|c|c|}
\hline \multirow{2}{*}{$\begin{array}{l}\text { Item } \\
\text { Learn- } \\
\text { ing } \\
\text { Trials }\end{array}$} & \multicolumn{8}{|c|}{ Category Learning Trials } \\
\hline & 1 & 2 & 3 & 4 & 5 & 6 & 7 & $\mathrm{~N}$ \\
\hline 1 & & & & 1 & & 1 & & \\
\hline 2 & 1 & 2 & & & & & 1 & 1 \\
\hline 3 & 1 & 2 & 3 & & 3 & & & 2 \\
\hline 4 & 6 & 1 & 1 & 1 & & 2 & 2 & 5 \\
\hline 5 & 2 & & 1 & 1 & 1 & 1 & & 7 \\
\hline 6 & 2 & 1 & 2 & 2 & 1 & 1 & 1 & 8 \\
\hline 7 & 1 & 1 & 1 & 2 & 3 & 2 & 4 & 16 \\
\hline 8 & 6 & 1 & & 3 & 4 & 7 & 2 & 26 \\
\hline 9 & 3 & & & 2 & 1 & 3 & 1 & 11 \\
\hline $\mathbf{N}$ & 18 & 5 & 6 & 17 & 12 & 24 & 10 & 181 \\
\hline
\end{tabular}

Note- $N$ indicates the number of unlearned responses. The sum of each column is the total number of category responses learned on that trial; the sum of each row is the total number of item responses learned on that trial. Each entry is the number of cases in which a category response and an item response were learned on that particular pair of trials.

item response was learned first for 12 faces, and both responses were learned on the same trial for 12 faces. Although the discrimination model did not predict faster category learning, these results are not inconsistent with a discrimination model, because less discrimination is required when some patterns share a common response. For example, one would not have to discriminate between confusable pairs of patterns if they shared the same response, and the faster learning of category responses may have been the result of confusable pairs' belonging to the same category (e.g., Faces 1 and 3). Also, Figure $3 b$ illustrates that, in contrast to the distributions shown in Figure 3a, there is considerable overlap between the category and item distributions. It is highly likely, therefore, that the superiority of category learning in Experiments 2 and 3 was the result of the discrimination demands of the task and not the result of the number of response alternatives.

\section{GENERAL DISCUSSION}

The results indicated that category learning occurred very rapidly, whereas item learning occurred very slowly, if at all. Furthermore, there appeared to be only a weak correlation between category learning and item learning when the two responses were given on separate trials. There was a nonsignificant correlation between the two kinds of learning, the ease of category learning varied greatly across the 10 schematic faces, whereas the ease of item learning was approximately the same, and the rate of category learning was unaffected by the substitution of item learning on alternate trials. The results suggest that the categories were learned very quickly on the basis of abstracted information (prototypes or distinctive features), whereas item leaming depended on the more difficult task of discriminating among the individual exemplars. When patterns were randomly assigned to categories and abstraction was not possible, category learning occurred at about the same rate as item learning.

The slow rate of item learning and the fact that only one-third of the item responses were learned suggest that subjects would have found it difficult to use the average distance or nearest neighbor strategy as a basis for classifying novel patterns. These strategies require that subjects be able to discriminate among the individual exemplars, a requirement that was not very well satisfied in the current experiments. This should not imply, however, that exemplar learning never occurs. Three recent papers (Brooks, 1978; Medin \& Schaffer, 1978; Robbins, Baresi, Compton, Furst, Russo, \& Smith, 1978) have emphasized the role of specific exemplars in determining people's classifications, and it is very likely that psychologists will continue to debate for some time the relative importance of abstract vs. exemplar-specific knowledge. Application of the current learning paradigm to other tasks may provide a more detailed analysis of this issue than would otherwise be possible.

There are two properties of categories that should influence the extent of prototype abstraction and exemplar learning. As the number of patterns within a category increases, prototype abstraction should improve (Homa, Cross, Cornell, Goldman, \& Shwartz, 1973), but exemplar learning should decrease because of the additional demands on memory. As the variability of patterns within a category is reduced, prototype abstraction should improve (Peterson, Meagher, Chait, \& Gillie, 1973), but exemplar learning should decrease because patterns are more difficult to discriminate. Thus Garner's (1962, p. 168) argument that pattern variability is neither good nor bad but depends on the particular performance criterion is clearly shown by its potential to produce opposite effects on category vs. item learning.

Variation in the physical similarity of category patterns is illustrated by the Rosch, Mervis, Gray, Johnson, and Boyes-Braem (1976) distinction between three levels of categories. The most general level, superordinate categories, includes categories such as musical instruments, fruit, tools, clothing, furniture, and vehicles. The next level, the basic level, includes categories such as pianos, apples, hammers, pants, lamps, and cars. The most specific level, subordinate categories, includes categories such as grand pianos, Mackintosh apples, claw hammers, Levis, desk lamps, and sports cars. Rosch et al. hypothesized that the basic level contains the most inclusive categories that can be identified from the averaged shapes of their members. According to the Rosch et al. hypothesis, there should be little gain in a subject's ability to form a prototype at a subordinate, rather than at a basic, level, but a large loss in a subject's 
ability to form a prototype at a superordinate level. Rosch et al. tested their hypothesis by asking subjects to identify outline shapes of objects that had been formed by averaging the shapes of category exemplars, normalized for size and orientation. Subjects correctly named 25 of the 32 prototypes at the basic level and did not do significantly better at the subordinate level. Performance at the superordinate level was not significantly better than chance. The results support the view that there is a basic level of abstraction at which an average of real-world shapes can be recognized.

The distinction between different levels of categorization provides a convenient point to return to the first issue raised in this paper: the difference between a discrimination theory (Gibson \& Gibson, 1955) and a categorization theory (Bruner, 1957) of perception. The discrimination theory emphasizes that perceptual learning occurs through discovering distinctive features that enable one to detect differences between objects that formerly looked alike. The categorization theory emphasizes that perceptual learning occurs through an act of induction: A new object is recognized when it can be fit into a familiar category. The distinction between different levels of categories suggests that the extent of discrimination, as opposed to generalization learning, should depend on the level at which learning occurs. As one proceeds from the subordinate level to the superordinate level, discrimination learning becomes less important and generalization learning becomes more important. The three-level division (subordinate, basic, and superordinate) provides a convenient hierarchy, although in some cases additional levels should be useful in specifying the extent of discrimination learning required to learn different exemplars. The subordinate distinction between a sports car and a four-door sedan would require less discrimination learning than identifying different models of cars, which in turn would require less discrimination learning than identifying different manufacturing years of the same model.

Since the basic level is intermediate in the hierarchy, it is at this level that there should be the greatest amount of interaction between discrimination and generalization leaming. Classifications higher in the hierarchy should require increasing amounts of induction relating physically different exemplars (hammer, saw, screwdriver), whereas classifications lower in the hierarchy should require increasing amounts of discrimination learning to distinguish between physically similar exemplars (ball hammer, claw hammer). The importance of the basic level of categorization (see the discussion by Rosch et al., 1976) may in part be the result of the ease with which objects can be categorized when an intermediate level of generalization and discrimination is required, rather than when either extreme is required.

In addition to the number and variability of exemplars within a category, the demands of the task should influence the extent of exemplar leaming. Brooks (1978) listed several factors that encourage storage of information about the individual members of a category. A child may need to know not only that an animal is a dog, but whether a particular dog is friendly. Leaming alot about individuals allows for more flexible use of information when there is uncertainty about future task demands. Even when learning about individuals occurs slowly, learning could occur because people may see the same individuals many times for every new instance that is encountered. Learning the individual members of a category may even be necessary when the basis for defining a category is not clear.

Another way in which the specific task may influence the classification rules used by people is by determining what kind of abstraction occurs when individual exemplars are not well learned. The weighted-features prototype model successfully predicted how people would learn and classify schematic faces. On the other hand, feature frequency models, such as the n-gram model, have been successfully applied to predict how subjects would classify patterns composed of geometric transformations (Neumann, 1974) and people into clubs (Hayes-Roth \& Hayes-Roth, 1977). Both these tasks used stimuli that could not be easily represented in a continuous multidimensional space. A useful objective of future research would be to identify how characteristics of the task influence people's classifications, since it is unlikely that a single strategy is used on all occasions. It is hoped that the discrimination. generalization paradigm will help to identify these strategies.

\section{REFERENCES}

Brooks, L. Nonanalytic concept formation and memory for instances. In E. Rosch \& B. Lloyd (Eds.), Cognition and categorization. Hillsdale, N.J: Lawrence Erlbaum, 1978.

BRUner, J. On perceptual readiness. Psychological Review, 1957, 64, 123-152.

GARNER, W. R. Uncertainty and structure as psychological concepts. New York: Wiley, 1962.

Gisson, E. Principles of perceptual learning and development. New York: Appleton, 1969.

Gibson, J. J., \& Gibson, E. Perceptual learning: Differentiation or enrichment? Psychological Review, 1955, 62, 32-41.

Hayes-Roth, B., \& Hayes-Roth, F. Concept learning and the recognition and classification of exemplars. Journal of Verbal Learning and Verbal Behavior, 1977, 16, 321-338.

Homa, D., Cross, J., Cornell, D., Goldman, D., \& Shwartz, S. Prototype abstraction and classification of new instances as a function of number of instances defining the prototype. Journal of Experimental Psychology, 1973, 101, 116-132.

Medin, D. L., \& Schaffer, M. M. Context theory of classification learning. Psychological Review, 1978, 85, 207-238.

Neumann, P. G. An attribute frequency model for the abstraction of prototypes. Memory \& Cognition, 1974, 2, 241-248.

Peterson, M. J., Meagher, R. B., Chait, H., \& Gillie, S. The abstraction and generalization of dot patterns. Cognitive Psychology, 1973, 4, 378-398. 
Prck, A. Improvement of visual and tactual form discrimination. Journal of Experimental Psychology, 1965, 69, 331-339.

Posner, M. I., \& Keele, S. W. On the genesis of abstract ideas. Journal of Experimental Psychoiogy, 1968, 77, 3, 353-363.

REED, S. K. Pattern recognition and categorization. Cognitive Psychology, 1972, 3, 382-407.

REED, S. K. Psychological processes in pattern recognition. New York: Academic Press, 1973.

Reed, S. K., \& Friedman, M. P. Perceptual vs. conceptual categorization, Memory \& Cognition, 1973, 1, 157-163.

Reitman, J. S., \& Bower, G. H. Storage and later recognition of exemplars of concepts. Cognitive Psychology, 1973, 4, 194-206.

Robbins, D., Barresi, J., Compton, P., Furst, A., Russo, M., \& Smith, M. A. The genesis and use of exemplar vs. prototype learning in abstract category learning. Memory \& Cognition, 1978, 6, 473-480.

Rosch, E., Mervis, C. B., Gray, W., Johnson, D., \& Boyes-BraEm, P. Basic objects in natural categories. Cognitive Psychology, 1976, 8, 382-440.

SebESTYEN, G. S. Decision-making processes in pattern recognition. New York: MacMillan, 1962.

THeios, J. Finite integer models for learning in individual subjects. Psychological Review, 1968, 75, 292-307.

\section{NOTES}

1. The issue of distinctive features learning vs. prototpye learning has been investigated using a paradigm developed by
Pick (1965). However, the "prototype" in this paradigm is more analogous to an unanalyzed template than to a central tendency abstracted from a category. This distinction is discussed in more detail by Reed (1973).

2. The average distance to the patterns is not identical to the distance to the average pattern because the distance metric is a nonlineas function of a pattern's attributes. The city block metric would be a linear function if it were not for the absolute value signs.

3 . The distance between the eyes and nose provides a good cue for differentiating between the two categories, although there were only a moderate number of references to this relation. I reanalyzed the previous classification data (Reed, 1972) to test the prediction that the greater the distance between the eyes and the nose, the greater the number of subjects who should classify the pattern into Category $B$. The rank-order correlation between the two variables was .58 when the category patterns were simultaneously presented (Reed, 1972, Problem 2A) and .56 when the category patterns were first learned (Reed, 1972 Problem 3). The corresponding correlations were .63 and .64 for predictions based on the physical distance to the category prototypes and were both .83 for predictions based on the psychologically scaled distances to the prototypes (Reed, 1972, Table 6).

(Revision accepted for publication September 5, 1978.) 